\title{
Effect of Lipoplex Charge Ratio on Transfection Efficiency of Osteosarcoma Cells
}

\author{
Mahshid Shokri ${ }^{1}$, Mahmood Tavallaie ${ }^{2 *}$, Seyed Mostafa Hosseini ${ }^{2}$ \\ ${ }^{1}$ Department of Biomedical Engineering, Science and Research Branch, Islamic Azad University, Tehran, Iran \\ ${ }^{2}$ Human Genetic Research Center, Baqiyatallah Medical Sciences University, Tehran, Iran
}

\begin{abstract}
Received: September 15, 2016; Accepted: September 30, 2016; Published: October 6, 2016
*Corresponding author: Mahmood Tavallaie, Human Genetic Research Centre, Baqiyatallah Medical Sciences University, Tehran, Iran, Tel: (+98) 26403292 ; Fax: (+98) 26413382; E-mail: tavalla.mah@gmail.com
\end{abstract}

\begin{abstract}
Summary
In recent years, liposomes are considered as a potential gene delivery vehicles. In order to increase transfection of cells by these types of carriers, many researchers have been tried to find relationships between factors related to liposome structure and efficiency of transfection process. In this study, the effect of the factor of charge ratio on transfection of osteosarcoma cells with pIRESEGFP gene is investigated. A liposomal formulation of DOTAP/DOPE/ Cholesterol was fabricated through lipid film hydration method. Morphology of liposomes was shown by Transmission electron microscopy. Lipoplexes were prepared by complexation of liposomes with DNA. Zeta potential of lipoplexes with different charge ratios (2.5:1, 2:1 and 1.5:1) was compared with liposome. Transfection of osteosarcoma cells with three ratios of lipoplexes was compared to Lipofectamine ${ }^{\mathrm{TM}}$. The results showed that the ratio of $(2: 1)$ is optimal for transfection of cancer cells while no significant difference was observed between formulations $(1.5: 1)$ and $(2.5: 1)$ by the end of the experiment $(p>0.05)$.
\end{abstract}

Keywords: Liposome; Transfection; Gene delivery systems; Osteosarcoma

\section{Introduction}

Gene therapy is an effective strategy for treatment of many genetic diseases such as malignant melanoma, cystic fibrosis and Gaucher's disease [1]. In this strategy, diseased cells are transfected with specific genes to acquire normal function [2,3].

Liposome-based gene delivery systems which were first reported by Felgner (1987) have been widely investigated as the most common non-viral delivery systems [1, 4-7]. Many cationic liposome formulations have been developed. The most ordinary phospholipids used in preparation of such liposomes include: phosphatidylcholine (PC), phosphatidylserine (PS), phosphatidylethanolamine (PE), phosphatidylinositol (PI), dipalmitoylphosphatidylcholine (DPPC), 1,2-dioleoyl-snglycero-3-phospho-L-serine, distearyl phosphatidylcholine (DSPC), dipalmitoyl phosphatidylserine, dipalmitoyl phosphatidylglycerol dioleoyl phosphatidylethanolamine (DOPE) [8].

Cationic liposomes have many advantages in gene delivery applications. Firstly, they can react spontaneously with negatively charged polynucleotides to form stable complexes which are able to preserve nucleic acids from degradation. Secondly, their cationic nature ensures the binding to negatively charged membrane of most of the cells via electrostatic interactions promoting entrance of genetic materials into cells through endocytosis $[2,4,9]$. Thirdly, pegylation of liposome surface for prolonged circulation or its modification with various functional groups such as specific targeting ligands or fusogenic peptides for endosomal escape could be helpful $[3,9]$.

Apart from these advantages, inadequate transfection efficiency of liposomes compared to viral vectors is problematic [10]. To ameliorate efficiency, in-depth exploration of mechanisms of gene delivery (i.e. complex formation, binding to cell membrane, endocytosis and then entry of DNA to nucleus by membrane receptors and finally expression of associated genes) in these systems is required $[1,4,9,11,12]$.

A number of factors determine the efficiency of transfection process. chemical structure of lipids, morphology of lipoplexes , liposome particle size the presence of helper lipids (such as dioleoyl phosphatidylethanolamine (DOPE) and cholesterol or dioleoyl phosphatidylcholine (DOPC) which are used to facilitate association of the DNA with the lipoplexes) are some of these factors $[1,4,11,13-16]$. Among them, Charge ratio (+/-) of the formed complexes is regarded as one of the most important factors of transfection efficiency. This factor which could be seen in terms of zeta potential is also important in determining the stability of lipoplex [1,4].

In this article, liposomal formulations of DOTAP/DOPE/ Cholesterol with different charge ratios were fabricated and their efficiency for transfection of osteosarcoma cells was investigated. Choice of DOPE was owing to its ability to reduce the cytotoxicity of cationic liposomes and improving transfection efficiency.

\section{Materials and methods}

\section{Materials}

1,2-di-O-octadecenyl-3-trimethylammonium propane (DOTAP) and 1,2-dioleoyl-sn-glycero-3 phosphoethanolamine (DOPE) 
were purchased from Avanti Polar lipids. Cholesterol and Sucrose were obtained from Sigma-Aldrich and Alfa Aesar, respectively. Murine osteosarcoma cell lines (CRL-2836 ${ }^{\mathrm{TM}}$ ) were obtained from American Type Culture Collection. Dulbecco's modified Eagle's medium (DMEM) with 10\% Fetal Bovine Serum (FBS) and penicillin-streptomycin solutions were purchased from GIBCO Inc. All the solvent were of analytical grade.

\section{Fabrication of liposomes and lipoplexes}

Lipid film hydration method was selected for fabrication of liposomes. At first, DOTAP/DOPE/cholesterol with a molar ratio of $1: 1: 2$ were dissolved in chloroform (total concentration of $5 \mathrm{mg} / \mathrm{ml}$ ). The resulting solution was heated to 40 o $\mathrm{C}$ for 6 hours in ambient pressure to remove the solvent. Then, the vacuum was applied for 24 hours to complete dryness of lipid films. The films then hydrated through $1 \mathrm{ml}$ of $15 \mathrm{mg} / \mathrm{ml}$ sucrose aqueous solution to obtain vesicles. Finally, lipoplexes of desired (+/-) charge ratio were prepared by mixing certain amounts of DNA and cationic liposomes and incubating for 20 minutes at room temperature.

\section{Surface charge of liposomes and lipoplexes}

Lipoplex formation and surface charge were studied using zeta potential analyzer (Brookhaven Instruments). All the measurements were performed at room temperature using water as the solvent.

\section{Morphology of liposomes}

The morphology of liposomes after reconstitution was observed employing Transmission Electron Microscopy (TEM, JEOL JEM-2100). After addition of water, a certain amount of vesicle suspension was poured on the TEM grid and negatively stained using $2 \%$ phosphotungstic acid solution. Imaging was carried out after removing an extra volume of suspension at the accelerating voltage of $200 \mathrm{kV}$.

\section{Cell Culture and transfection assay}

The transfection efficiency of formulations and the effect of (+/-) charge ratio on the transfection process of cancer cells were investigated. Murine osteosarcoma cells were cultured in complete DMEM with a final concentration of $10 \%$ FBS and $1 \%$ penicillin, streptomycin and antimycotic. At a value of confluency of $80 \%$, cells were transfected with cationic liposome-DNA (pIRES-EGFP) complexes at different charge ratios (fig 1) while Lipofectamine $^{\mathrm{TM}}$ reagent-DNA complex was used as the positive control. Prior to transfection, media in each well was replaced with serum-free DMEM and changed to media with serum after 24 hours incubation. Plates were analyzed for green fluorescence at certain time intervals using an Essen Bioscience Incucyte Zoom system.

\section{Statistical analysis}

Two-tailed Student's t-test was used for the test of statistical significance using Microsoft Excel 2007 software (Microsoft, Redmond, WA). Calculating $p$-value, the level of the presumption against null hypothesis was determined for every experiment.

\section{Results}

\section{Zeta potential of liposomes and lipoplexes}

Fig 1 compares zeta potential of liposomes before and after DNA loading.

\section{Morphology of liposomes}

Reconstitution of vesicles occurs through the addition of pure water to the powder. Water diffusion toward the core of vesicles might be induced by osmotic pressure generated by sucrose encapsulated inside the vesicles. Morphology of reconstituted liposomes could be observed through TEM images as illustrated in Fig 2. As it shown in this figure, vesicles are spherical with an average size of around $75 \mathrm{~nm}$.

\section{Transfection efficiency}

The (+/-) charge ratios of 1.5:1, 2:1 and 2.5:1 all containing $1 \mu \mathrm{g}$ DNA were used to transfect osteosarcoma cells. In this case, Lipofectamine $^{\mathrm{TM}}$ reagent was used as the positive control. Fig 3 shows the percentage of transfected cells for lipoplexes with different charge ratios during the first 44 hours. The percent of transfected cells is increasing with time indicating the dynamic transfection process within the test interval. However,

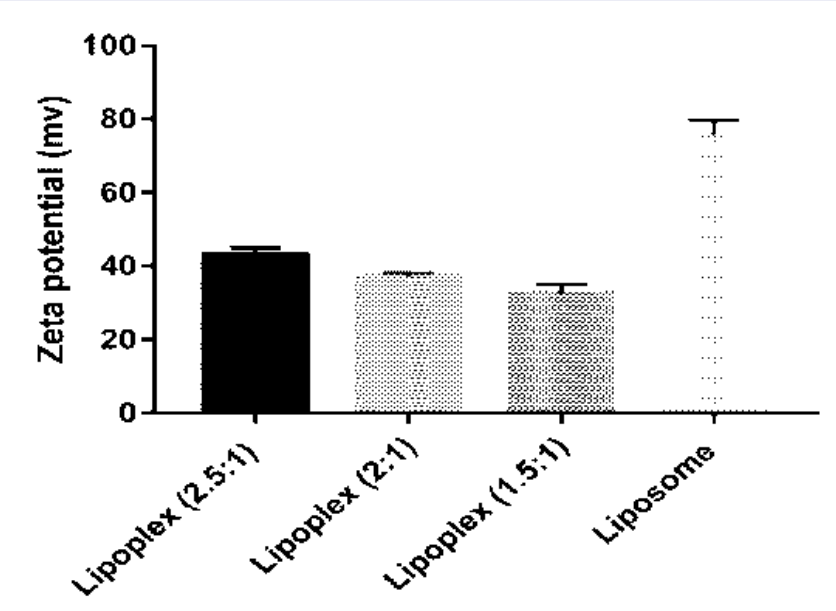

Figure 1: Zeta potential of liposome and lipoplexes with three different charge ratios $(2.5: 1,2: 1$ and $1,5: 1)$

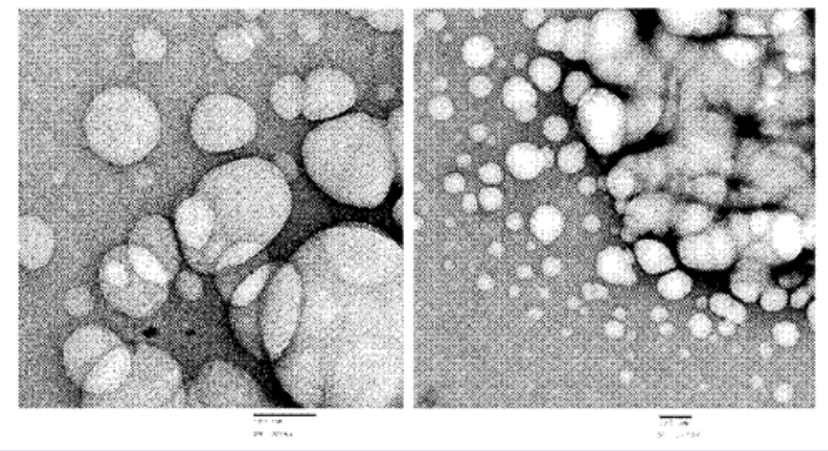

Figure 2 : TEM images of liposome after reconstitution. 
less than $5 \%$ of the cells were transfected after 44 hours even using Lipofectamine ${ }^{\mathrm{TM}}$ as the transfection reagent. It seems that osteosarcoma cells generally resist being transfected by liposomal formulations [17].

Among all, no significant difference was observed between formulations (1.5:1) and (2.5:1) by the end of the experiment ( $p>$ $0.05)$. Other formulations also were the same in the first 30 hours $(p>0.05)$ but significant difference appeared between each two after this period $(p<0.05)$. The observation demonstrates the importance of sufficiently prolonged experiments to distinguish efficiency of different gene delivery systems.

Plotting $p$-values against time for each group was performed (Fig 4). The $p$-value was a periodic function of time with several extremes. At maximum points, formulations operate more similarly while at minimums, formulations function differently from each other.

\section{Discussion}

Cationic liposomes of DOTAP/DOPE/Cholesterol were

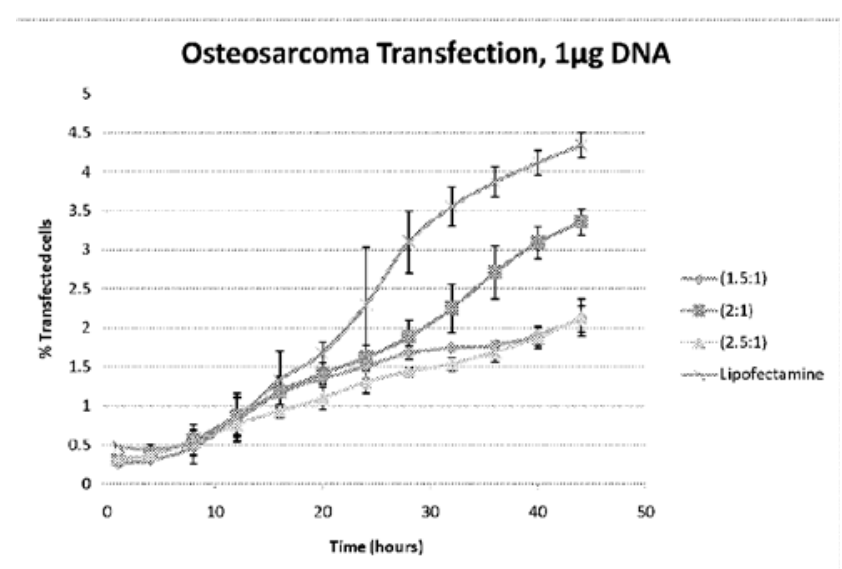

Figure 3: Effect of charge ratio on the transfection of osteosarcoma cells by vesicles

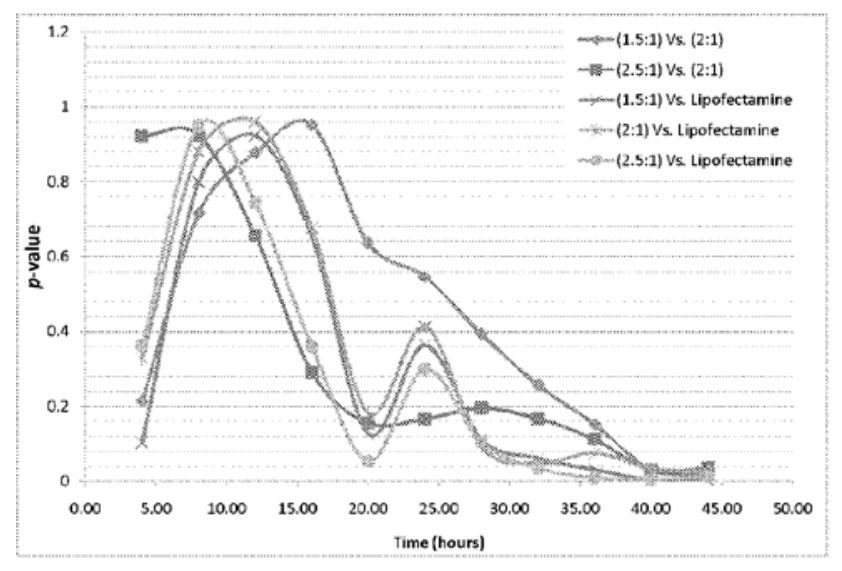

Figure 4: $p$-values of different transfection experiment groups versus time prepared through a dry lipid film method. In this method, vesicles are produced via self-assembly of amphiphilic lipids in aqueous media. It is known that cellular uptake of liposomes is under the influence of its physicochemical properties [18]. The charge of vesicles is one of the most significant factors. Zeta potential measurements showed that vesicles are positively charged because of the presence of amine groups of cationic lipid, DOTAP. The value of zeta potential decreased after the incorporation of negatively charged DNA molecule. Therefore, fig 1 supports the formation of lipoplexes. The net positive charge of the formulations is favorable for transfection of cells since complexation of lipoplexes with negatively charged cell membrane is facilitated due to electrostatic interactions and DNA can enter the cells via adsorptive endocytosis $[7,19]$.

In terms of morphology, liposomes have spherical shape confirming reconstitution of them from the powder. Vesicles with various sizes could be observed in the TEM figures because of polydisperse particle size distribution of the samples.

It has been shown that the cell type, the gene used for transfection, the type of helper lipid, and DNA-/lipid+ charge ratio, all affect transfection efficiency in a statistically significant manner [20]. Saito et al. found that the apparent cellular uptake may increase as the (+/-) charge ratio increases but the transfection efficiency was reduced at high charge ratios. They suggested that in achieving a high efficiency of transfection, intracellular trafficking is the more important process rather than the degree of uptake which is achieved in an optimal charge ratio [21]. As could be seen, in this study the highest transfection efficiency was seen in the case of 2:1 charge ratio. Some authors have reported an optimum (+/-) charge ratio for lipoplexes by which the cells are best transfected $[18,22,23]$ agreeing with our results. This means neither highly positive vesicles nor neutral ones can transfect the cells like partially positive vesicles do.

Additionally, cell cycle influences the transfection process. Mortimer et al. monitored the transfection process of human ovarian carcinoma tumor cells using lipoplexes over time for both synchronized and unsynchronized cells [24]. Their data suggests that even for unsynchronized cells, transfection boosts at certain time points. It is the reason why $p$-values are a periodic function of time in Figure 4.

In summary, In this study the effect of charge ratio on transfection of osteosarcoma cells was investigated. Three different charge ratios of lipoplexes $(2.5: 1,2: 1$ and 1.5:1) were compared with liposome in terms of surface charge. Decreased values of positive charge of three formulations compared to liposome suggest the formation of lipoplexes. Transfection of osteosarcoma cells with three ratios of lipoplexes was compared to Lipofectamine ${ }^{\mathrm{TM}}$. The results suggest that the ratio of $(2: 1)$ is optimal among two other ratios which show that surface charge is not the only effective factor in gene transfection of cells and there is an optimal range of charge ratios in which the efficiency of transfection is in the highest amount.

\section{References}

1. Ma B, Zhang S, Jiang H, Zhao B, Lv H. Lipoplex morphologies and their influences on transfection efficiency in gene delivery. Journal of Controlled Release. 2007;123(3):184-194. 
2. Ciani L, Ristori S, Salvati A, Calamai L, Martini G. DOTAP/DOPE and DCChol/DOPE lipoplexes for gene delivery: zeta potential measurements and electron spin resonance spectra. Biochimica et Biophysica Acta (BBA) - Biomembranes. 2004;1664(1):70-79.

3. Zhang Y, Satterlee A, Huang L. In Vivo Gene Delivery by Nonviral Vectors: Overcoming Hurdles? Molecular Therapy. 2012;20(7):12981304. DoI:10.1038/mt.2012.79Tros de Ilarduya C, Sun Y, Düzgüneş N. Gene delivery by lipoplexes and polyplexes. European Journal of Pharmaceutical Sciences. 2010;40(3):159-170. doi: 10.1016/j.ejps.

4. Maurer N, Mori A, Palmer L, Monck MA, Mok KW, Mui B, et al. Lipidbased systems for the intracellular delivery of genetic drugs. Molecular membrane biology. 1999;16(1):129-140.

5. Yagi K, Noda H, Kurono M, Ohishi N. Efficient gene transfer with less cytotoxicity by means of cationic multilamellar liposomes. Biochemical and biophysical research communications. 1993;196(3):1042-1048.

6. Felgner PL, Gadek TR, Holm M, Roman R, Chan HW, Wenz M, et al. Lipofection: a highly efficient, lipid-mediated DNA-transfection procedure. Proceedings of the National Academy of Sciences of the United States of America. 1987;84(21):7413-7417.

7. Popovska 0. An Overview: Methods for Preparation and Characterization of Liposomes as Drug Delivery Systems2014.

8. Nayerossadat N, Maedeh T, Ali PA. Viral and nonviral delivery systems for gene delivery. Advanced Biomedical Research. 2012;1:27. doi: 10.4103/2277-9175.98152

9. Cevher E, Çağlar EŞ, Sezer AD. Gene Delivery Systems: Recent Progress in Viral and Non-Viral Therapy. 2012 ;:. DOI: 10.5772/53392

10. Simberg D, Weisman S, Talmon Y, Barenholz Y. DOTAP (and Other Cationic Lipids): Chemistry, Biophysics, and Transfection. 2004;21(4):257-317.

11. Felgner JH, Kumar R, Sridhar C, Wheeler CJ, Tsai YJ, Border R, et al Enhanced gene delivery and mechanism studies with a novel series of cationic lipid formulations. Journal of Biological Chemistry. 1994;269(4):2550-2561.

12. Kerner M, Meyuhas O, Hirsch-Lerner D, Rosen LJ, Min Z, Barenholz Y. Interplay in lipoplexes between type of pDNA promoter and lipid composition determines transfection efficiency of human growth hormone in NIH3T3 cells in culture. Biochimica et Biophysica Acta (BBA)-Molecular and Cell Biology of Lipids. 2001;1532(1):128-136.

13. Pozzi D, Marchini C, Cardarelli F, Amenitsch H, Garulli C, Bifone A, et al. Transfection efficiency boost of cholesterol-containing lipoplexes. Biochimica et Biophysica Acta (BBA)-Biomembranes. 2012;1818(9):2335-2343. doi:10.1016/j.bbamem.

14.Zuhorn IS, Bakowsky U, Polushkin E, Visser WH, Stuart MCA, Engberts JBFN, et al. Nonbilayer phase of lipoplex-membrane mixture determines endosomal escape of genetic cargo and transfection efficiency. Mol Ther. 2005;11(5):801-810. DOI:10.1016/j. ymthe.2004.12.018

15. Ross $P$, Hui S. Lipoplex size is a major determinant of in vitro lipofection efficiency. Gene therapy. 1999;6(4):651-659. DOI:10.1038/ sj.gt.3300863

16. kenhirao T, Kubo T, Kazuhikoigarashi Kt, Murakami T, Yasunaga Y, Ochi M. Targeted gene delivery to human osteosarcoma cells with magnetic cationic liposomes under a magnetic field. International journal of oncology. 2003;22(5):1065-1071.

17. Radwan Almofti M, Harashima H, Shinohara Y, Almofti A, Baba Y, Kiwada H. Cationic liposome-mediated gene delivery: biophysical study and mechanism of internalization. Archives of biochemistry and biophysics. 2003;410(2):246-253.

18. Lasic DD. Liposomes in gene delivery: CRC press; 1997;ISBN 9780849331091 - CAT\# 3109.

19. Boktov J, Hirsch-Lerner D, Barenholz Y. Characterization of the interplay between the main factors contributing to lipoplexmediated transfection in cell cultures. The journal of gene medicine. 2007;9(10):884-893. DOI:10.1002/jgm.1079

20. Saito Y, Kawakami S, Yabe Y, Yamashita F, Hashida M. Intracellular trafficking is the important process that determines the optimal charge ratio on transfection by galactosylated lipoplex in HEPG2 cells. Biological and Pharmaceutical Bulletin. 2006;29(9):1986-1990.

21. Maitani Y, Igarashi S, Sato M, Hattori Y. Cationic liposome (DCChol/DOPE = 1: 2) and a modified ethanol injection method to prepare liposomes, increased gene expression. International journal of pharmaceutics. 2007;342(1):33-39. DOI: 10.1016/j. ijpharm.2007.04.035

22. Jellema RK, Bomans P, Deckers N, Ungethum L, Reutelingsperger CP, Hofstra L, et al. Transfection efficiency of lipoplexes for site-directed delivery. Journal of liposome research. 2010;20(3):258-267. doi: 10.3109/08982100903384137.

23. Mortimer I, Tam P, MacLachlan I, Graham R, Saravolac E, Joshi P. Cationic lipid-mediated transfection of cells in culture requires mitotic activity. Gene therapy. 1999;6(3):403-411. 